\title{
The Influence of Evolutionary Psychology on Human Mating and Sexual Behavior
}

\author{
Nida Mohammad Fayyaz \\ Department of Psychology \& Counselling, College of Humanities and Social Sciences, United Arab Emirates University, \\ United Arab Emirates
}

Received March 16, 2019; Revised April 17, 2019; Accepted May 3, 2019

Copyright $(2019$ by authors, all rights reserved. Authors agree that this article remains permanently open access under the terms of the Creative Commons Attribution License 4.0 International License

\begin{abstract}
Over the course of history, human beings have taken keen interest in the mating activity. The survival of the human species has been dependent on sexual mating and human reproduction; the need for sexual companionship has played a pivotal role in building societies and establishing social as well as intimate relationships. To overcome the problem of finding a suitable sexual partner, men and women adopt different strategies, based on their roles in the society. This paper discusses the impact of Evolutionary Psychology on Human mating and sexual behavior. David Buss's Sexual Strategies Theory is considered one of the major research programs to investigate how this relationship works. This paper aims to provide an emotional and cultural perspective on how evolutionary psychology has influenced human sexual and mating behaviors. It can be said that Buss's theory, despite being one of its kind, has failed to look at the complete picture. These limitations are due to various reasons; such as over emphasis on the polygamous nature of men, the evaluation of parental investment of both sexes, the problems each of the sexes can solve by using these strategies and how mating is considered an individual activity instead of a cooperative one. The cultural, philosophical and emotional take on the evolution of human sexual behavior brings a fresh and creative outlook.
\end{abstract}

Keywords Sexual Strategies Theory, Mating, Sexual Behavior, Evolution, Psychology, Culture

"Immortality which makes love a universal feature. Thus, the universal human desire is not for beauty, but it is for everlasting goodness and happiness." Diotima of Mantinea, Greek Philosopher. (Murphy, 1997)

"Aristophanes describes how Hephaistos could meld two bodies together for all eternity to satisfy human desire, and yet even this did not satisfy humans because sexual intercourse is not what humans are truly after. Aristophanes suggests that humans seek the mythic wholeness that sex approximates." (Evans, 2006)

"The heart has its reasons which reason know nothing of." Blaise Pascal, Mathematician. (Haselton \& Ketelaar, 2005)

\section{Introduction}

The world around us has been divided into various societies and cultures. Despite their countless differences, human beings in these societies are bound by their innate need to form relationships and engage in mating behavior. To successfully fulfil this need, human beings tend to adopt different strategies, either long-term or short-term. The variations in human mating and sexual behavior can also be witnessed in our ancestral societies. Thus, the topic of human mating and sexual behavior has garnered significant attention. From Philosophers to Psychologists, most minds have wandered to find answers and to come up with explanations to understand and predict this aspect of human behavior (Irigaray \& Carlston, 1989; Buss \& Schmitt, 1993; Buss, 1995; Evans, 2006; Schulz, 2009; Williams \& Jacobson, 2016). The most common perspective, however, involves Evolutionary Psychology and its impact on human mating and sexual behavior.

The basis of Evolutionary Psychology stems from the combination of modern evolutionary theory, biology, cognitive psychology, anthropology, computer science and Paleo archaeology (Buss \& Schmitt, 2011). The main purpose of evolutionary psychology is to study human behavior as being developed over time into evolved psychological mechanisms (Confer et. al, 2010). This approach has been successful in explaining human sexual behavior as it is based on the notion that the mind includes many mental adaptations which is the product of natural and sexual selection operations, deep rooted into human 
evolution (Haselton \& Ketelaar, 2005). In other words, our current sexual behavior has evolved into a more sophisticated system of mate selection, yet holds the same set of adaptive problems. Since these are problems of different nature, human beings have developed an abundance of complex and varied emotions; this helps them deal with adaptive problems (Barret 2005; Symons 1979).

This paper will discuss the Evolutionary Perspective on Human Mating and Sexual Behavior. To further understand this complex yet intriguing phenomena, it will analyze David Buss's Sexual Strategies Theory (SST). The theory is considered one of the most influential evolutionary psychological research programs and is pivotal in studying human psychology and the mind (Buss, 1989, 1998, 2003, 2007; Buss \& Schmitt, 1993). Despite its significant role in understanding mating and sexual behavior, it has also been highly controversial. The theory has been criticized for Buss's interpretation of his empirical studies and the presumptions (ideological and fundamental) behind these propositions (Schulz, 2009; Buller 2005). Furthermore, this study attempts to identify other potentially important view-points, which were omitted by Buss, such as the impact of emotions and culture.

\section{Sexual Strategies Theory}

The theory begins with the idea that mating is a universally common activity, which results in formal alliances between men and women. The idea of marriage is regarded as a formal reproductive alliance between men and women; this grants them sexual access, mutual presumably long-term commitment between the two and expectations of child-rearing activity and legitimacy of the offspring. Since there is no guarantee of the duration of mating relationships, they may last for a few hours, days, months or even years. This idea further facilitates the two types of mating Buss mentions: short-term mating and long-term mating. In his theory, Buss makes numerous claims such as:

1) Lifelong mating with a single person does not appear to be the norm for humans.

2) Mating strategies are context dependent however the principles that govern the mating of women and men are different.

3) In human evolutionary history, men and women have pursued short-term and long-term mating strategies. As different adaptive problems occur with each type of mating, they need to be resolved to be a success.

4) Levels of parental investment differ between the sexes. This affects the mating strategy as men prefer short-term mating as compared to women.

5) As the goal of all sexual relationships is to produce offspring, men are constrained in their reproductive success by the number of fertile women they can inseminate. They face multiple problems; such as problem of how many partners, identifying sexually accessible women and the problem of minimizing commitment and investment.

6) The problem with identifying women who are reproductively valuable and ensuring certainty in paternity. This includes finding women with good parenting skills and women who are able and willing to commit long-term.

7) The reproductive constraints lead to adaptive problems which women had to solve themselves; i.e. problem of identifying men who can invest resources, the problem of identifying men who are willing to invest resources in her and the offspring, the problem of identifying men with good parenting skills, men willing and able to commit to a long-term relationship and men who are willing to protect her.

8) Different evolved sexual strategies for both men and women adopted to counter these issues.

Based on the above-mentioned points, the Sexual Strategies Theory is recognized as a standard evolutionary psychological paradigm to explain human mating and sexual behavior. However, as there are two sides to every coin, we must also recognize the limitations of the theory. Despite coming up with empirical evidence that supports his argument, Buss's theory can easily be considered a victim of hasty generalization. In other words, it is an attempt to paint everyone with the same brush. Different adaptive problems require different adaptive solutions. We cannot have a "general solution" as there are no "general problems" (Buss, 1995).

\section{Critique of the Sexual Strategies Theory}

While the theory is based on the promiscuousness of men, it completely rejects the idea of monogamy. It should be noted that this contradicts the theory's stance on long-term mating (Schulz, 2009). This brings us to the evolved concept of Monogamy: a long and essentially exclusive mating relationship between one male and one female. Cheating may impact the relationship dynamics, but it does not negate the existence of monogamy (Wittenberger \& Tilson, 1980). We, human beings, have been involved in the evolution of monogamy. It is said that monogamy has evolved, placing more emphasis on male care. In a monogamous mating situation, the male-partner has the potential to raise his offspring and make sure that they will survive. Interestingly, monogamy has also affected the brain size of humans compared to other mammals as fathers have also evolved in their ability to hunt and scavenge meat. By doing this, they ensure nutrient supply and sufficient caloric availability for their children.

Monogamy can also be witnessed in social insects, in terms of Eusocial behavior, as mating with a single male is 
ancestral for all the eight independent eusocial lineages. Thus, producing concrete evidence that monogamy was critical in the evolution of eusociality. This also supports the prediction of inclusive fitness theory (Hughes, et al., 2008). Even though there is evidence of the existence of polygamy in our ancestral societies, one cannot disregard the significant impact of monogamy on our current mating behavior. This is because females who indulge in monogamous mating are likely to obtain benefits which may not be available otherwise. Also, they ensure that their mates do not desert them. Monogamous mating is significant in ensuring territorial marking as it is more beneficial to mate with an unmated available male as compared to mating with an already mated male (Wittenberger \& Tilson, 1980). Hence, the promiscuousness of men is likely to be a problem in the current mating scenario.

In addition to that, human mating and sexual activity is a cooperative venture so it is essential to consider both partners as a team. However, Buss considers both sexes independently (Schulz, 2009). As mating activity cannot happen individually, it is important to consider the strategy of males and females to predict the success of the strategies adopted. Another significant cause for concern is Buss's assumption that male promiscuity is the only strategy adopted for successful mating encounters, when men could easily adopt strategies that can be based on care and need for long-term affection (Schulz, 2009).

Another criticism of the theory would be the lack of consideration of the environment and the impact of culture on mating and sexual behavior.

\section{Human Evolution and Mating Behavior}

Throughout human history, successful reproductive activity has been pivotal to human evolution. Charles Darwin proposed in the Sexual Selection Theory- which operates with intrasexual competition between members of one sex with the loser typically failing to mate and intersexual selection (also called female choice) making member of one sex discriminatory about whom they mated with- that men and women operate on different cognitive biases when it comes to human mating (Buss, 2019). This theory, put forward by Darwin, support Buss's claims of the difference in the sexual strategies operated by males and females. In his empirical study, Buss has been able to successfully prove that males are likely to feel threatened by the members of their own gender whereas females are more selective in mate selection.

Despite evolutionary behavior supporting the aforementioned notions, most researchers disregard the idea that lifetime monogamy is not a norm for human behavior. Long before the evolution of modern human beings (i.e. Homo Sapiens), cooperative breeding and monogamous human mating existed in parts of ancient Africa. Anthropological evidence suggests that Neanderthals and Homo Erectus civilizations were actively involved in monogamous mating. Before their migration to Europe, Neanderthals indulged in monogamous mating within their clan. The males would impregnate the female and then to ensure lineage, the female was protected by the male. Furthermore, as there was no way to find out if the offspring was interbred, the pregnant female was unable to change clans or move away from the vicinity. However, after their migration to Europe, it is widely believed that they mated with the modern humans resulting in interbreeding (Wade, 2010; Williams, 2013). As for the species of Homo-Erectus, monogamy seems to be the norm as this allows mates to receive extensive reproductive from kin and clan members. Whereas Homo-sapiens evolved to monogamy as a result of environmental factors (Fletcher et al., 2015).

Even though considerable research has been done to deny Buss's claims on monogamy, it is also important to mention that historically, Australopithecus afarensis were polygynous- suggesting that one male would indulge in reproductive activities with more than one female. One reason for this could be that they had a shorter period of growing and reached adulthood faster than modern humans. This resulted in less parental investment which meant reproductive constraints, for both males and females, were relatively minimum (Smithsonian National Museum of Natural History, 2016). However, Australopithecus afarensis gradually shifted their mating behavior from polygamy to monogamy during the Homo Erectus era (Fletcher et al., 2015).

Modern human beings have evolved into monogamous mates; this could be attributed to the biological features of male sexual organs and reproductive behavior. Contrary to Buss's stance that men are more inclined towards pursuing short-term mating strategies, research suggests that human males are fit for monogamous mating or polygynous primates but not multi-male/female mating arrangements (Fletcher et al., 2015). Competition between men to fertilize a woman's egg(s) exists, to gain access to reproductive value of females, which is known as Sperm Competition (Shackelford et al., 2007). The existence of Sperm Competition suggests that the human male recognizes his limitation, when it comes to gaining access to the reproductive abilities of a female, specifically because of his biological makeup. As mentioned by (Fletcher et al., 2015), human males have smaller testes with less sperm production, slower replacement of sperm, less vigorous sperm, lower sperm quality and lower ejaculation rates. This is in comparison to their other male counterparts, such as in Chimpanzees. Monogamy reduces the problem of Sperm Competition (Shackelford et al., 2007), thus resulting in a shift towards long-term mating strategy. 


\section{Cultural impact on Human Mating and Sexual Behavior}

To understand the implications of culture of human mating and sexual behavior, in terms of evolutionary psychology, one needs to come up with the definition that identifies with the cause. Culture, as defined by Jahoda (2012), are artefacts which is the material word that has been modified over history to adapt to human thought and action. It also lays the down the foundation for human development, as it comprises of successful and adaptive participation in human activities. This development aids human beings for interaction with other people; culture is additionally responsible for preserving and transmitting social norms, beliefs, conventions, etc.

Considering the above-mentioned definition, it can easily be implied that culture plays a vital role in our interaction with other people. Since human mating and sexual activity requires social interaction, it would be naïve to disregard the role of culture in shaping these interactions. Social intelligence and cooperative abilities are facilitated by long-term interpersonal relationships (Fletcher et al., 2015). Homo Sapiens (i.e. the modern human beings) have evolved to patrilocal mating which resulted in social monogamy (Williams, 2013). The Sexual Strategies theory states that a male is likely to pursue various strategies to successfully mate with a female; as women are likely to require long-term commitment (sexual and emotional), therefore, men need to modify their mating strategies to cater to these wants. In other words, females have pressured males into adopting long-term mating strategies (Wlodarski, Manning \& Dunbar, 2015).

In this context, let us examine the differences between matriarchal and patriarchal society. In cultures, such as the Tibetan Buddhists called Mosuo, where women can have multiple sexual partners and the children belong to the mothers. The biological fathers live with their mothers and are brought up by their maternal families (Booth, 2017). This could also be witnessed in the post-colonial African societies. Religious influences have significantly impacted the dynamics of family life; i.e. shifting it towards a more patriarchal society (Amadiume, 2006). As matriarchal societies acknowledge the kinship of women and the offspring is expected to live in the grandmother's house. This is because women are greatly respected since they give birth. Furthermore, in matriarchal societies, no one gender is given more importance than the other as both are responsible for bringing life (Gottner - Abendroth, 2004). Few of the key concepts of Buss's theory can be considered flawed as it claims that women require men to protect them and their offspring and women may not be interested in short-term copulation.

Despite the widespread idea that modern human beings have evolved into monogamous societies, it would be naïve to disregard those cultures where polygamy is a social norm. In $82 \%$ of cultures, polygamy is permitted and men can have social and sexual relationships with more than one female (Fletcher et al., 2015). Polygamy is relatively high in Muslim Communities of West Africa and common in Arab nations even Non-Muslim Majority countries, such as Israel. Polygamy is legal in South Africa, Egypt, Eretria, Morocco, Malaysia, United Arab Emirates and Saudi Arabia (Vallely, 2010). There are multiple reasons for this; such as religion, access to multiple women's reproductive abilities, continuing the family name, etc. As mentioned by Hayase \& Liaw (1997), most women get married at an early age to men who are older; this ensures early exposure to pregnancy and expand reproductive duration. There is a high proportion of adult polygamous males who are single; the sexual desires of these men are satisfied by the wives of their other male relatives. Thus, it could be said that Buss (1993) was not wrong when he claimed that men are likely to be promiscuous in their mating behavior and that women are likely to engage in copulation with men who "provide for them" and are willing to invest resources in the union.

Finally, it can be stated that culture plays a vital role in the sexual strategy being adopted. It is also important to understand that the dominant marital arrangements usually involve monogamous mating. For example, in western cultures, polygamy is considered "outmost offence against chastity and marriage" (A. Cannon, 1974). On the contrary, in eastern cultures, polygamy is a mark of high social, economic or political status (Krenawi, 1997). Based on these arguments, it is clear that when the modern human beings adopted cultures and evolved into civilized societies, the presence of cultural factors were crucial in assessing and pursuing mating strategies.

\section{Influence of Emotions on Human Mating Behavior}

The concept of true love and soulmates have existed long before the study of Psychology has. In the past, philosophers like Socrates, Plato, Aristophanes and Diotima have shown a great deal of interest in the romantic and emotional aspects of human sexual relationships (Jayne, 1985; A Bloom, 1993; Murphy 1997). Emotions, such as jealousy, love or even fear, are vital when studying any kind of human behavior, especially sexual and mating behavior.

As mating and reproduction comes with a significant adaptive challenge, it is not surprising that we use more than one psychological mechanism to overcome those challenges. This allows us to form many emotions which provide a solid base for decision making. Emotions affect everything; including the way we think and behave in social and personal settings (Clore, Schwarz \& Conway, 1994). Love and guilt are considered vital in social decision-making, especially when it comes to evolutionary psychology of emotions (Haselton \& Ketelaar, 2005). Per the Commitment-device theory (Frank, 1988), the effects 
of guilt in relationships may influence emotions, such as lust, to make decisions which do not result in immediate gratification but reaps long term benefits (Haselton \& Ketelar, 2005). The rewards and punishment concept further paves the way towards understanding this. Precognition- the ability of a person to understand that present decisions and/or responses may influence an individual's future- is vital when it comes to understanding how people may not settle for short-term sexual encounters and look for something more meaningful and enduring (Bem DJ, 2011).

Another important aspect to consider is how certain emotions may change the strategy being followed or result in overall changes in strategic goal (i.e. instead of focusing on the reproductive value of mates, other aspects may be considered). Certain emotions may change our responsiveness to cues; such as fear or arousal. This results in differences in terms of optimal mating strategy but in most context, exclusive coupling would have been beneficial (Pillsworth \& Haselton, 2005). Buss (1993) completely omits the idea of love as a driving force in terms of sexual context. As Haselton \& Ketelaar (2005) writes, love changes how people perceive sexual relationships. Anyone who claims to be in love is likely to not find anyone else attractive except their own partner. This will lead to long-term commitment and avoid infidelity as sexual temptations are blocked. In other words, love is a vital force in terms of copulation and how it affects commitment in a relationship, resulting in a monogamous relationship.

Romantic love is a motivational force when it comes to monogamy and long-term mating. There is historical evidence that our ancestors used to adopt different strategies to compete against each other and to retain mate for a longer period of time; thus, ensuring maximum reproductive gains, security of the offspring and certainty of lineage. This can also be seen in the Sperm Competition phenomena (Shackelford et al., 2007). Romantic love involves attachment, caregiving and sex. Cognitive biases operate which allow monogamous mating and reduce the likelihood of infidelity and/or polygamy. Even in cultures where polygamy is allowed, romantic love does not allow primates to find other mates. In cases where male promiscuousness remains a strong force, conflict between partners, jealousy, insecurities and perceived threat may exist. In order to ensure that Homo Sapiens (i.e. the modern human beings) evolved into a civilized society, the mating behavior had to shift towards monogamy instead of polygamy (Fletcher et al., 2015).

\section{Limitations and Recommendations}

A major limitation of this paper is that it is not an empirical study, as no population was studied and no investigation was conducted, the study is based on theoretical framework. Since the influence of evolutionary psychology on human mating and sexual behavior is based on anthropology, paleo-archeology and evolutionary psychology, it is difficult to successfully measure the influence. Hence, qualitative comparisons were made instead of quantitative ones. Another limitation of this study involves insufficient data collection and lack of empirical evidence. Most researchers have conducted theoretical analysis of the phenomena instead of an empirical investigation. Finally, as there are gaps in the archaeological and anthropological investigation of human evolution, there are ambiguities when it comes to evolution and mating behavior.

Based on the arguments stated in this paper, it is crucial to conduct empirical research to understand if there is a correlational and/or causation relationship between the evolutionary theory and human mating and sexual behavior. Furthermore, the relationship of culture and emotions with human mating and sexual behavior needs to be examined. As human beings are social beings, it would be necessary to consider how social interactions in different cultures influence the norms of human sexual behavior. Lastly, as most of the research is conducted on Western population, it would be beneficial to broaden the scope of the study, which would pave the way for further understanding of how culture and emotions influence human mating behavior, specifically from Evolutionary Psychology perspective.

\section{Conclusions}

Despite Buss's numerous somewhat successful attempts to explain sexual strategies and human sexual behavior, we can easily find limitations in his explanation. This includes how fidelity is a very strong concept and men may be inclined to keep one mate, while going against their basic instinct, as they have done so in many earlier works. It is also important to identify the role of emotions and culture, especially when it comes to how people choose their sexual mates. While long-term and short-term strategies may vary, it is important to consider the reproductive activity based on teamwork. To be successful, both parties need to come up with compatible strategies.

As Buss emphasis on the role of males in terms of reproduction and then being the sole-provider for the females and their children, he omits the existence of (few) matriarchal societies. The societies in which women are responsible for looking after their children. This view results in difference of opinion, especially when it comes to the motivations of women when they look for sexual encounters.

All in all, the theory lacks a strong argument in favor of the strategies explained. As with many other theories, this could be an acceptable explanation of human sexual behavior merely because there are no other 
empirical-evidence-based theories. However, there is a strong need to understand how emotions and culture influence the Sexual Strategies Theory and Human Sexual Behavior.

\section{REFERENCES}

[1] Al-Krenawi, A., Graham, J. R., \& Al-Krenawi, S. (1997). Social work practice with polygamous families. Child and Adolescent Social Work Journal, 14(6), 445-458.

[2] Amadiume, I. (2006). Sexuality, African religio-cultural traditions and modernity: Expanding the lens. Codesria bulletin, 1(2), 26-28

[3] Barrett, R. D., MacLean, R. C., \& Bell, G. (2005). Experimental evolution of Pseudomonas fluorescens in simple and complex environments. The American Naturalist, 166(4), 470-480.

[4] Bem, D. J. (2011). Feeling the future: experimental evidence for anomalous retroactive influences on cognition and affect. Journal of personality and social psychology, 100(3), 407

[5] Bloom, A. (1993). Love and friendship.

[6] Buller, D. J. (2005). Evolutionary psychology: the emperor's new paradigm. Trends in cognitive sciences, $9(6)$, 277-283.

[7] Buss (2019), Evolutionary Psychology- The Science of the Minds, $6^{\text {th }}$ Edition, 28-40.

[8] Buss (1995), Psychological Sex Differences: Origins through sexual selection, 164 .

[9] Buss \& Schmitt (1993): Sexual Strategies Theory: An Evolutionary Perspective on Human Mating, Psychological Review, 100 (2), 204.

[10] Buss \& Schmitt (2011), Evolutionary Psychology and Feminism, Sex roles, 64 (9-10), 786

[11] Buss, D. M. (1989). Sex differences in human mate preferences: Evolutionary hypotheses tested in 37 cultures. Behavioral and brain sciences, 12(1), 1-14

[12] Buss, D. M. (2007). The evolution of human mating. Acta Psychologica Sinica, 39(3), 502-512.

[13] Buss, D. M., \& Reeve, H. K. (2003). Evolutionary psychology and developmental dynamics: comment on Lickliter and Honeycutt (2003).

[14] Buss, D. M., Haselton, M. G., Shackelford, T. K., Bleske, A L., \& Wakefield, J. C. (1998). Adaptations, exaptations, and spandrels. American psychologist, 53(5), 533.

[15] Cannon, C. A. (1974). The Awesome Power of Sex: The Polemical Campaign against Mormon Polygamy. The Pacific Historical Review, 61-82.

[16] Clore, G. L., Schwarz, N., \& Conway, M. (1994). Affective causes and consequences of social information processing. Handbook of social cognition, 1, 323-417.

[17] Confer, J. C., Easton, J. A., Fleischman, D. S., Goetz, C. D.,
Lewis, D. M., Perilloux, C., \& Buss, D. M. (2010). Evolutionary psychology: Controversies, questions, prospects, and limitations. American Psychologist, 65(2), 110.

[18] Evans (2006): Diotima and Demeter as Mystagogues in Plato's Symposium, Hypatia, 21 (2), 1-27.

[19] Ficino, M., \& Jayne, S. R. (1985). Commentary on Plato's Symposium on Love.

[20] Fletcher, G. J., Simpson, J. A., Campbell, L., \& Overall, N. C. (2015). Pair-bonding, romantic love, and evolution: The curious case of Homo sapiens. Perspectives on Psychological Science, 10(1), 20-36.

[21] Frank, R. H. (1988). Passions within reason: the strategic role of the emotions. WW Norton \& Co.

[22] Göttner-Abendroth, H. (2004). Matriarchal society: Definition and theory. Vaughan, Genevieve (Hg.): The Gift, A Feminist Analysis. Rome: Athanor, S, 69-80.

[23] Hannah Booth: The Kingdom of women: the society where a man is never the boss. The Guardian.

[24] Haselton \& Ketelaar (2005): Irrational Emotions or Emotional Wisdom? The Evolutionary Psychology of affect and social behavior. Affect in social thinking and behavior, $8,21$.

[25] Hayase, Y., \& Liaw, K. L. (1997). Factors on polygamy in Sub-Saharan Africa: Findings based on the demographic and health surveys. The Developing Economies, 35(3), 293-327.

[26] Hughes, W. O., Oldroyd, B. P., Beekman, M., \& Ratnieks, F. L. (2008). Ancestral monogamy shows kin selection is key to the evolution of eusociality. Science, 320(5880), 1213-1216.

[27] Irigaray \& Carlstone (1989), The language of man, Cultural Critique, 13, 191-202.

[28] Jahoda, G. (2012). Critical reflections on some recent definitions of "culture". Culture \& Psychology, 18(3), 289-303.

[29] Murphy (1997): Analysis of Love: Diotima and Freud (205a-206a), Deakin University, Melbourne Australia.

[30] Pillsworth, E. G., \& Haselton, M. G. (2006). Male sexual attractiveness predicts differential ovulatory shifts in female extra-pair attraction and male mate retention. Evolution and human behavior, 27(4), 247-258.

[31] Schulz (2010): It takes two: Sexual strategies and game theory, Studies in History and Philosophy of Biological and Biomedical Sciences, 41 (1), 41-49.

[32] Shackelford, T. K., Goetz, A. T., McKibbin, W. F., \& Starratt, V. G. (2007). Absence makes the adaptations grow fonder: Proportion of time apart from partner, male sexual psychology, and sperm competition in humans (Homo sapiens). Journal of Comparative Psychology, 121(2), 214.

[33] Smithsonian National Museum of Natural History (2016), What does it mean to be human?

[34] Symons, D. (1979). The evolution of human sexuality.

[35] Wade (2010), Signs of Neanderthals Mating with Humans, 
New York Times,

[36] Williams \& Jacobson (2016); Effects of copulins on rating of female attractiveness, mate-guarding and self-perceived sexual desirability, Evolutionary Psychology, 14(2), 1474704916643328

[37] Williams, Ruth (2013), The Mating Habits of Early Hominins, The Scientist.

[38] Wittenberger, J. F., \& Tilson, R. L. (1980). The evolution of monogamy: hypotheses and evidence. Annual review of ecology and systematics, 11(1), 197-232.

[39] Wlodarski, R., Manning, J., \& Dunbar, R. I. M. (2015). Stay or stray? Evidence for alternative mating strategy phenotypes in both men and women. Biology letters, 11(2), 20140977.

[40] Vallely (2010), The Big Question: What is the history of polygamy, and how a problem is it in Africa? The Independent. 